\title{
Temporal Dynamics of Fusarium virguliforme Colonization of Soybean Roots
}

\author{
Jie Wang, Janette L. Jacobs, Mitchell G. Roth, and Martin I. Chilvers, ${ }^{\dagger}$ Department of Plant, Soil and Microbial Sciences, Michigan State \\ University, East Lansing, MI 48824
}

\begin{abstract}
Soybean sudden death syndrome (SDS) caused by Fusarium virguliforme is one of the most yield limiting soybean diseases in the United States. SDS disease symptoms include root rot and foliar symptoms induced by fungal toxins. Soybean cultivar resistance is one of the most effective SDS disease management options, but no cultivar displays complete resistance. Soybean SDS foliar symptoms are the primary phenotype used to screen and breed for SDS resistance. Root rot or root colonization measures are seldom utilized, partly due to the lack of convenient and accurate methods for quantification of $F$. virguliforme. In this study, greenhouse and field experiments were conducted to determine the temporal dynamics of $F$. virguliforme colonization of soybean roots using quantitative real-time PCR (qPCR). The infection coefficient (IC), or ratio of $F$. virguliforme DNA to soybean DNA, was determined in soybean cultivars with different SDS foliar resistance ratings. In greenhouse experiments, $F$. virguliforme was detected in all cultivars 7 days after planting (DAP), with a peak in IC at 14 DAP. All soybean cultivars

developed SDS foliar symptoms, but $F$. virguliforme soybean root colonization levels did not significantly correlate with SDS foliar symptom severity. In field experiments, SDS foliar symptoms developed among soybean cultivars in alignment with provided foliar resistance ratings; however, the $F$. virguliforme IC were not significantly different between SDS foliar symptomatic and asymptomatic cultivars. F. virguliforme was detected in all cultivars at the first sample collection point 25 DAP (V3 vegetative growth stage), and the IC increased throughout the season, peaking at the last sample collection point 153 DAP (postharvest). Collectively, appearance and disease severity ratings of SDS foliar symptoms were not associated with $F$. virguliforme quantity in roots, suggesting a need to include $F$. virguliforme root colonization in breeding efforts to screen soybean germplasm for $F$. virguliforme root infection resistance. The findings also demonstrates root colonization of the pathogen on nonsymptomatic soybean cultivars leading to persistence of the pathogen in the field, and possible hidden yield loss.
\end{abstract}

The primary causal agent of soybean sudden death syndrome (SDS) in North America is the soilborne fungus Fusarium virguliforme (Aoki et al. 2003). F. virguliforme causes both root rot and foliar leaf chlorosis and necrosis. Foliar SDS symptoms are a result of phytotoxins produced by $F$. virguliforme in the roots; the fungus has never been recovered from aboveground tissues (Rupe 1989). SDS root infection and foliar symptom development are not simultaneous. Early in the growing season, $F$. virguliforme conidia or chlamydospores germinate and form germ tubes to infect soybean roots, and these infections have been detected as early as 18 days after planting under field conditions (Gao et al. 2006; Njiti et al. 1997). In a greenhouse experiment, Huang and Hartman (1998) recovered F. virguliforme from roots within 10 days after planting. The onset of SDS foliar symptoms typically occurs at early reproductive stages (R1 beginning of flowering) (Fehr et al. 1971; Roy et al. 1997). Fungal toxins produced by $F$. virguliforme are translocated from the roots to the leaves through the xylem (Abeysekara and Bhattacharyya 2014). It was proposed that early infection of the radical roots at the seedling stage is necessary for $F$. virguliforme penetration into xylem tissues in order for foliar symptom development (GongoraCanul and Leandro 2011a; Navi and Yang 2008). Root infection and root rot in the absence of foliar symptoms may also result in hidden yield loss, and is difficult to evaluate.

${ }^{\dagger}$ Corresponding author: M. I. Chilvers; E-mail: chilvers@msu.edu

Funding: We would like to thank the Michigan Soybean Promotion Committee, North Central Soybean Research Program, the Carter Harrison Endowed Graduate Student Fund, and Pioneer DuPont for funding.

*The $\boldsymbol{e}$-Xtra logo stands for "electronic extra" and indicates that five supplementary figures are published online.

Accepted for publication 17 June 2018.

This article is in the public domain and not copyrightable. It may be freely reprinted with customary crediting of the source. The American Phytopathological Society, 2019.
Unfavorable conditions for soybean seed germination and emergence, such as low temperature, high precipitation, high soil moisture, and compacted soils may increase the risk of infection by $F$. virguliforme, by prolonging the germination process (Gongora-Canul and Leandro 2011b; Scherm and Yang 1996). To avoid these unfavorable conditions during germination or emergence, delayed planting has been suggested as a possible SDS management strategy (Hershman et al. 1990; Roy et al. 1997); however, there are often yield penalties associated with late planting, and the short window for field operations means that this is often not a viable management option (Kandel et al. 2016).

SDS foliar symptoms have been extensively used as a phenotype in soybean breeding programs for evaluating soybean germplasm for resistance (Clark et al. 2013; Njiti et al. 1996; Wen et al. 2014). However, it has been demonstrated that SDS foliar resistance and root infection are controlled by separate loci (Kazi et al. 2008). Evaluation of soybean root rot resistance to $F$. virguliforme has primarily relied on visual rating of percent root surface discoloration (Ortiz-Ribbing and Eastburn 2004; Srour et al. 2012), and evaluation of root infection has been determined via the number of $F$. virguliforme colony forming units (CFU) (Luo et al. 1999; Njiti et al. 1997). However, these methods are difficult to reproduce across labs and may be inaccurate in quantification of $F$. virguliforme.

The relationship between SDS foliar symptom development and severity and $F$. virguliforme level of infection is not clear. Two root disease-rating methods (i.e., visual rating of percent root discoloration and counting CFU of $F$. virguliforme from root tissues) have been utilized to screen for soybean root resistance to $F$. virguliforme infection (Gongora-Canul et al. 2012; Njiti et al. 1997). The culturebased quantification method provides a good estimation of viable $F$. virguliforme in soybean roots or soil samples, but it is often inaccurate, as $F$. virguliforme colony growth on culture medium may be out-grown by fast growing fungi. Additionally, the $F$. virguliforme colony morphology is not distinct from other closely related fungal species (Cho et al. 2001; Njiti et al. 1997). The visual estimation of root surface discoloration can be unspecific and subjective, as the root surface discoloration may be caused by other soil-borne soybean pathogens, such as other Fusarium spp., Pythium spp., Rhizoctonia solani, or Phytophthora spp. (Arias et al. 2013; Dorrance et al. 
2003), which makes this method difficult to reproduce between labs. The low sensitivity and nonspecific nature of the culture-based or visual rating methods can limit the interpretation of the root disease ratings. With increased interest in breeding soybeans that are resistant to root infection by $F$. virguliforme, there is a critical need to develop an accurate and reproducible root disease or infection rating method to evaluate resistance to $F$. virguliforme.

Quantitative real-time PCR (qPCR) provides an alternative method to the culture-based or visual rating methods. qPCR has been widely used to diagnose and quantify plant pathogens in soil or plant tissues (Bilodeau et al. 2012; Chilvers et al. 2007; Hughes et al. 2009). There are several qPCR assays that have been developed for the quantification of $F$. virguliforme in plant tissues or soil (Gao et al. 2004; Li et al. 2008; Wang et al. 2015; Westphal et al. 2014). The performance of the $F$. virguliforme specific assays have been compared, and each assay showed distinct variations in regard to specificity and sensitivity, with assays developed based on the rDNA intergenic spacer region demonstrating the greatest potential for $F$. virguliforme quantification (Kandel et al. 2015). Based on the performance related to specificity, sensitivity, and consistency, the qPCR assay developed by Wang et al. (2015) was used in this study.

We hypothesized that quantities of $F$. virguliforme in soybean root tissue would correlate to foliar symptoms, and set out to improve the understanding of the temporal dynamics of $F$. virguliforme root colonization. The objectives of this study were to i) evaluate the colonization dynamics of $F$. virguliforme with $\mathrm{qPCR}$ in soybean root tissues throughout the growing season; ii) determine if there were root colonization differences among soybean cultivars; and iii) determine the relationship between SDS foliar symptom development and root colonization by $F$. virguliforme.

\section{Materials and Methods}

$F$. virguliforme inoculum preparation. $F$. virguliforme isolates (VB2a and Mont-1 = NRRL 22292) were transferred onto Nash Snyder (NS) medium (Leslie et al. 2008) and incubated at room temperature for 2-3 weeks to allow full-plate colonization. Sorghum (milo maize) seeds were used as a growth substrate for $F$. virguliforme inoculum. Sorghum seeds were soaked overnight in deionized water, drained of excess water, and $1.8 \mathrm{~kg}$ of seeds weighed into mushroom spawn bags (Fungi Perfecti, Olympia, WA). Sorghum seeds were autoclaved at $121^{\circ} \mathrm{C}, 18 \mathrm{psi}$ for $8 \mathrm{~h}$ and cooled to room temperature for $24 \mathrm{~h}$. Five $F$. virguliforme colonized NS medium plates, five noncolonized NS medium plates, and $500 \mathrm{ml}$ sterile deionized water were placed in a sterile stainless-steel blender carafe and homogenized at low speed for $30 \mathrm{~s}$. The homogenized inoculum slurry was evenly distributed across five mushroom bags containing autoclaved sorghum seeds, and bags were sealed with a heat sealer. Inoculum bags were incubated at room temperature with ambient light for 30 days and mixed by shaking every other day. After sorghum grains were completely colonized, the inoculum grains were spread onto brown kraft paper in trays and air-dried at room temperature with the aid of a fan for 3-4 days. $F$. virguliforme inoculum $\mathrm{CFU}$ were determined to be $6 \times 10^{9} \mathrm{CFU} / \mathrm{g}$ using serial dilution and plating onto Nash-Snyder medium.
Greenhouse experimental design. Greenhouse experiments were conducted to study the temporal dynamics of $F$. virguliforme colonization of soybean roots over a 5-week period. Plants were destructively sampled at weekly time points to quantify $F$. virguliforme DNA via qPCR. Four soybean cultivars (Asgrow lines AG2107 and AG2002, and Pioneer lines 92M82 and 92Y53) were selected based on their commercial SDS foliar disease resistance rating from susceptible to moderately resistant (Table 1 ). The greenhouse protocol was modified from the SDS inoculated-layer technique developed by R. Bowen and G. Hartman (personal communication). A soil mix was prepared by homogenizing pasteurized sandy loam soil and SUREMIX (Sure, Galesburg, MI) at a ratio of 1:2. Trays (10.2 $\times$ $35.5 \times 50.8 \mathrm{~cm}$, catalog number: 14-3401, Hummert, Earth City, $\mathrm{MO})$ with 16,5 -mm-diameter holes drilled into the bottom for drainage were used to perform the inoculum-layer method trial. Two paper towels were placed on the bottom of the tray to prevent soil mix loss, and 3.2 liters of soil mix was placed over the paper towels and gently leveled. A second layer consisting of homogenized soil and $F$. virguliforme inoculum (1.26 liters soil mix, $336 \mathrm{ml}$ inoculum) was added evenly across the tray. An additional 3.2 liters of soil mix was spread over the inoculum layer. Seven furrows were created across the width of each tray, equally spaced $6.35 \mathrm{~mm}$ apart. Two replicate trays were planted for each of the five time points. Each of the seven furrows was planted with three cultivar replicates, which consisted of five seeds per replicate. Each cultivar was replicated three times within a tray and planted in a completely randomized design, and the experiment was repeated twice. Finally, the seeds were uniformly covered with 1.6 liters of soil mix. Foliar symptoms of SDS were assessed at 35 days after planting (DAP), using the disease severity rating system prepared by R. Bowen and G. Hartman (2011, unpublished). A disease score of 1 indicates a healthy plant without foliar symptoms; scores 2 to 5 indicate development of leaf interveinal chlorosis followed by necrosis with increased percentage of leaf area affected receiving higher scores; scores 6 to 9 indicate symptoms of defoliation and premature death (note that this is a different scale from that used in the field). Root rot symptoms of SDS were assessed by estimating the percentage of discoloration and rot on the plant root system with a rating scale from 0 to $100 \%$.

Roots of 5 soybean plants were collected, washed under tap water to remove soil, oven-dried at $50^{\circ} \mathrm{C}$ for $48 \mathrm{~h}$, and root dry weight was determined. Soybean roots taken at V2 growth stage were dried and ground in FASTPREP tubes (MPBIO, Solon, OH) with the aid of a ceramic sphere (MPBIO) and five 2-mm glass beads. Roots collected after the V2 growth stage were ground using a coffee grinder (item number: 072-08-1562, KRUPS). To avoid cross contamination between samples, the inside of the coffee grinder was rinsed with $70 \%$ ethanol and wiped dry with paper towel. DNA was extracted with an AutoGenprep 850 Alpha system (AutoGen, Holliston, MA). One hundred milligrams of dried root tissue and $1 \mathrm{ml}$ plant lysis solution (AutoGen) were added to each sample tube. Tubes were sealed with aluminum foil and incubated in a water bath for $1.5 \mathrm{~h}$ at $75^{\circ} \mathrm{C}$. Preprocessed samples were submitted to the Michigan State University genomics core for automated high-throughput phenol chloroform DNA purification. The precipitated DNA pellet was dissolved in $150 \mu \mathrm{l} 1 \times$ Tris-EDTA buffer. DNA was quantified using

Table 1. Soybean cultivars used in this study

\begin{tabular}{lclll}
\hline Varieties & Relative maturity group & SCN resistance rating & SDS resistance rating & \multicolumn{1}{c}{ Experiment $^{\mathbf{b}}$} \\
\hline AG2107 & 2.1 & PI88788 & Susceptible & Greenhouse and field 2012 \\
AG2002 & 2.0 & PI88788 & Moderately resistant & Greenhouse and field 2012 \\
P92M82 & 2.8 & None & Susceptible & Greenhouse and field 2012 \\
P92Y53 & 2.5 & Peking (PI548402) & Moderately resistant & Greenhouse, field 2012, and field 2014 \\
P92Y11 & 2.1 & Peking (PI548402) & Susceptible & Field 2014 \\
P92Y51 & 2.5 & PI88788 & Moderately resistant & Field 2014 \\
P92Y60 & 2.6 & PI88788 & Susceptible & Field 2014 \\
P93M11 & 3.1 & None & Moderately resistant & Field 2014 \\
\hline
\end{tabular}

a Based on seed industry sudden death syndrome (SDS) susceptibility rankings on seed labels.

b Soybean cultivars used for research experiments. 
the Quant-iT dsDNA high-sensitivity assay kit (Invitrogen, Carlsbad, CA) on a 96-well SAFIRE microplate reader (TECAN, Männedorf, Switzerland).

Field experimental design. In 2012, field experiments were conducted at two locations: a grower's field near Decatur, MI in a Spinks loamy sand naturally infested with $F$. virguliforme and soybean cyst nematode; and a research field at Michigan State University (MSU) Agronomy Farm in East Lansing, MI in a Capac loam soil artificially inoculated with $F$. virguliforme at $6.6 \mathrm{ml} / \mathrm{m}(2 \mathrm{ml} / \mathrm{ft})$. The same four soybean cultivars used in the greenhouse study were planted at both field locations in a randomized complete block design with five replicated plots (Table 1). At the Decatur site, soybean plots were planted in

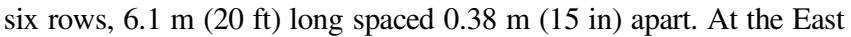
Lansing site, soybean plots were planted in two rows, $9.1 \mathrm{~m}(30 \mathrm{ft})$ long and spaced $0.76 \mathrm{~m}$ (30 in) apart. Planting dates were May 7 and May 11, 2012 at Decatur and East Lansing, respectively. In 2014, a field experiment was conducted at the Decatur site with five soybean cultivars (Table 1), replicated five times across 6-row plots in a randomized complete block design as in 2012, planted on May 5. The outer rows (i.e., first and sixth) were used for destructive root sampling, while the inner four rows were kept for harvest to estimate yield.

Each plot was rated for SDS disease severity (DS) and disease incidence (DI) based on a previously reported scoring method (Gibson et al. 1994). The DS scale ranged from 0 to 9 as described above. Each plot was rated by estimating the DS of symptomatic plants per plot, disease incidence was determined by estimating the percentage (0 to $100 \%)$ of SDS symptomatic plants in each plot. A disease index (DX) was derived by combining both DS and DI values to include both severity and incidence of foliar symptoms present in a soybean plot; DX was calculated using the formula: DX $=(D I \times$ DS)/9, where 9 represents the highest SDS disease severity rating possible. As opposed to the categorical DS rating scale, the DX scale is continuous ranging from 0 to 100. In 2012, 15 roots were collected from each field plot per cultivar at five time points from June 4 to October 9, 2012. Roots were dug with a shovel, and aboveground tissues were removed. Roots were transported to the lab, rinsed under tap water to remove attached soil and debris, and oven-dried at $50^{\circ} \mathrm{C}$ for $48 \mathrm{~h}$. Root dry weights per plot were measured. Dried soybean roots were ground in a Wiley mill (Thomas Scientific, Swedesboro, $\mathrm{NJ}$ ) with a $2-\mathrm{mm}$ pore size mesh screen. DNA was extracted as described above.

In 2014, to gain a better understanding of the relationship between $F$. virguliforme colonization and foliar symptom expression, the severity of SDS foliar symptoms and root rot were rated on individual plants, in addition to plot-wide rating. Fifteen soybean plants were sampled for each plot at four time points. Root tissue was processed for DNA extraction as described above.

Quantification of $\boldsymbol{F}$. virguliforme in soybean roots. The IC of $F$. virguliforme to soybean DNA ratio was calculated by conducting two separate qPCR assays. Following Wang et al. (2015), the qPCR master mix for $F$. virguliforme consisted of $10 \mu \mathrm{l}$ TaqMan Universal real-time PCR master mix (2X) (Applied Biosystems), $0.5 \mu 1$ TaqMan dual labeled MGB probe FvPrb3 $(10 \mu \mathrm{M}), 0.5 \mu$ l of each primer
(20 $\mu \mathrm{M}$, F6-3 and R6), $0.4 \mu \mathrm{l}$ bovine serum albumin $(10 \mathrm{mg} / \mathrm{ml})$, $0.6 \mu \mathrm{l}$ of HHIC-F primer $(20 \mu \mathrm{M}), 0.2$ of HHIC-R primer $(20 \mu \mathrm{M}), 0.4 \mu \mathrm{l}$ of HHIC-prb probe $(10 \mu \mathrm{M}), 0.5 \mu$ l linearized HHIC DNA plasmid (10 fg/ $\mu \mathrm{l}$ ) (Table 2) (Haudenshield and Hartman 2011), $4.4 \mu \mathrm{l}$ molecular grade water (Gibco, Carlsbad, CA) and $2 \mu \mathrm{l}$ DNA template (1:10 dilution with $\mathrm{dH}_{2} \mathrm{O}$ ). The HHIC exogenous control was added to the $F$. virguliforme assay to identify false negative reactions due to the presence of qPCR inhibitors. A $20-\mu l$ total reaction volume and two technical replicates were performed under the following qPCR cycling conditions: initialization at $50^{\circ} \mathrm{C}$ for $2 \mathrm{~min}$, one cycle at $95^{\circ} \mathrm{C}$ for $10 \mathrm{~min}$ for denaturing, and 40 cycles at $95^{\circ} \mathrm{C}$ for $15 \mathrm{~s}$ and $60^{\circ} \mathrm{C}$ for $1 \mathrm{~min}$ on an $\mathrm{ABI}$ StepOnePlus thermal cycler (Applied Biosystems, Carlsbad, CA).

A qPCR assay for quantification of soybean genomic DNA was designed on a single-copy beta-tubulin gene from the soybean genome. The qPCR master mix for the soybean quantification assay consisted of $10 \mu \mathrm{l}$ TaqMan Universal real-time PCR master mix (2X) (Applied Biosystems), $0.5 \mu 1$ TaqMan dual labeled MGB probe TUB-Prb-1 $(10 \mu \mathrm{M}), 0.5 \mu \mathrm{l}$ for each primer $(20 \mu \mathrm{M}$, TUB-F1 and TUB-R1) (Table 2), $0.4 \mu \mathrm{l}$ bovine serum albumin $(10 \mathrm{mg} / \mathrm{ml}$ ), $6.1 \mu \mathrm{l}$ of molecular grade $\mathrm{dH}_{2} \mathrm{O}$, and $2 \mu \mathrm{l}$ DNA template $(1: 100$ dilution with $\mathrm{dH}_{2} \mathrm{O}$ ).

PCR efficiency for both quantification assays was determined by running a five-point standard curve ranging from $1 \mathrm{ng}$ to $100 \mathrm{fg}$ of genomic DNA quantified with a fluorimeter. The qPCR conditions for the soybean genomic DNA quantification assay were optimized to be the same as the $F$. virguliforme $\mathrm{qPCR}$ assay, which makes it possible to run both assays on the same 96-well plate. Soybean DNA was quantified to create an $\mathrm{IC}$, a ratio of $F$. virguliforme and soybean $\mathrm{Ct}$ values, which is a normalized $F$. virguliforme infection value of soybean root tissue (Valsesia et al. 2005). The IC is calculated using the formula: $\mathrm{CT}_{\mathrm{Soy}} / \mathrm{CT}_{\mathrm{Fv}}$, where $\mathrm{CT}_{\mathrm{Soy}}$ is the cycle threshold of soybean DNA from the qPCR assay and $\mathrm{CT}_{\mathrm{Fv}}$ is the cycle threshold of the $F$. virguliforme qPCR assay. DNA dilution factors were accounted for in data manipulation prior to analyses.

Data analysis. Real-time qPCR data were collected from the realtime PCR thermal cycler using StepOnePlus software v2.3 (Applied Biosystems). Analysis of variance (ANOVA) was performed using the "stat" package in R v3.2 (R Core Team 2015), and soybean cultivar was used as a fixed effect to test for level of significance. Figures were generated using "graphic" and "ggplot2" packages (Wickham 2009). The area under infection coefficient curves (AUICC) were calculated using the function "audpc" in "agricolae" package (De Mendiburu 2014) in R v3.2 (R Core Team 2015).

\section{Results}

Greenhouse temporal dynamics of $\boldsymbol{F}$. virguliforme colonization. In the greenhouse experiments, $F$. virguliforme DNA was detected in the roots of all soybean cultivars at the first sampling time point, 7 DAP. In the first greenhouse experiment, the average IC ranged between 0.96 and 1.12 at $7 \mathrm{DAP}$, with the highest ratio detected at the second sampling time point (14 DAP), with an IC of 1.19 (Fig. 1). All subsequent sampling time points $(21,28$, and 35 DAP) had lower

Table 2. Primers and probes used in this study

\begin{tabular}{|c|c|c|c|c|}
\hline Primer names & Sequences (w/modifications) & Final concentration & Target & Source \\
\hline F6-3 & GTAAGTGAGATTTAGTCTAGGGTAGGTGAC & $500 \mathrm{nM}$ & rDNA-IGS ${ }^{\mathrm{a}}$ & Wang et al. 2015 \\
\hline R6 & GGGACCACCTACCCTACACCTACT & $500 \mathrm{nM}$ & rDNA-IGS $^{\mathrm{a}}$ & Wang et al. 2015 \\
\hline FvPrb3 & 6FAM-TTTGGTCTAGGGTAGGCCG-MGBNFQ & $250 \mathrm{nM}$ & rDNA-IGS ${ }^{a}$ & Wang et al. 2015 \\
\hline TUB-F1 & GCGGTGCTCATGGATCTAGAG & $500 \mathrm{nM}$ & Beta-tubulin $^{\mathrm{b}}$ & This study \\
\hline TUB-R1 & TGACCGTAGGGACCAGATCTG & $500 \mathrm{nM}$ & Beta-tubulin ${ }^{b}$ & This study \\
\hline TUB-Prb-1 & NED-AGGGACCATGGACAGC-MGBNFQ & $250 \mathrm{nM}$ & Beta-tubulin $^{\mathrm{b}}$ & This study \\
\hline HHIC-F & CTAGGACGAGAACTCCCACAT & $600 \mathrm{nM}$ & pJSH-B14c & Haudenshield and Hartman 2011 \\
\hline HHIC-R & CAATCAGCGGGTGTTTCA & $200 \mathrm{nM}$ & pJSH-B14c & Haudenshield and Hartman 2011 \\
\hline HHIC-Prb & 5HEX-TCGGTGTTGATGTTTGCCATGGT-3IABkFQ & $200 \mathrm{nM}$ & pJSH-B $14^{\mathrm{c}}$ & Haudenshield and Hartman 2011 \\
\hline
\end{tabular}

\footnotetext{
a Targets $F$. virguliforme.

b Targets soybean.
}

${ }^{c}$ Targets HHIC plasmid. 
ICs ranging between 0.99 and 1.14. The ANOVA test indicated that ICs of $F$. virguliforme were significantly different among the four cultivars at $28 \mathrm{DAP}$ and $35 \mathrm{DAP}(P<0.01)$, though the ICs of $F$. virguliforme were not always higher in the susceptible cultivar (i.e., AG2107 and 92M82, Fig. 1A). All soybean cultivars developed foliar symptoms by $35 \mathrm{DAP}$ (Fig. 1C), and foliar disease indices were not significantly different among the four cultivars $(P=0.73$, Fig. $1 C)$.

In the second greenhouse experiment, the ICs of $F$. virguliforme were higher than in the first greenhouse experiment. At 7 DAP, the average IC ranged between 1.42 and 1.46 . The mean $F$. virguliforme IC from 7 to 14 DAP increased from 1.43 to 2.02. In subsequent sampling time points $(21,28$, and 35 DAP), the $F$. virguliforme IC fluctuated between 1.78 and 2.15 . In the first four sampling time points, the $F$. virguliforme IC did not show significant differences among the four soybean cultivars $(P>0.05)$. At 35 DAP, both susceptible cultivars had significantly higher ICs than the moderately resistant cultivars $(P<0.01$, Fig. 1B). All four soybean cultivars developed SDS foliar symptoms by 35 DAP, and SDS foliar symptom disease indices were not significantly different among cultivars $(P=0.87$, Fig. $1 C)$.

To evaluate the level of colonization by $F$. virguliforme over time, the AUICC were calculated for each cultivar. The overall AUICC in the second greenhouse experiment were greater than the AUICC in the first greenhouse experiment $(P<0.01)$ for all cultivars. Within each greenhouse experiment, there were no significant differences among four cultivars $(P>0.7$, Fig. 1A and B). In the first greenhouse experiment, root dry weight increased over time and reached an average weight of $0.08 \mathrm{~g} /$ plant at $35 \mathrm{DAP}$, except for cultivar 92M82, which was significantly lower at $0.02 \mathrm{~g} /$ plant (Supplementary Fig. $\mathrm{S} 1$ ). In the second greenhouse experiment, root dry weight increased over time, but did not reach the same levels as in the first experiment. By 35 DAP, the average root dry weight was $0.03 \mathrm{~g} / \mathrm{plant}$. Significant root dry weight differences were observed among the four cultivars at 14,21 , and 28 DAP, but there were no significant root dry weight differences among cultivars at time point 35 DAP. Greenhouse results of root rot severity were variable between experiments (data not shown); surprisingly, susceptible cultivar 92M82 demonstrated significantly lower root rot than other cultivars in the first experiment. Correlation between greenhouse IC and root rot was significant $(P=0.0038)$ but weak ( $r=0.27$ ) using Spearman correlation (data not shown).

$F$. virguliforme colonization of field grown soybean roots. In 2012, F. virguliforme root colonization was comparable between the two field locations. Variation (standard deviation) in $F$. virguliforme ICs among soybean cultivars was greater at the East Lansing site than the Decatur site (Fig. 2A and B). F. virguliforme was
A

First greenhouse experiment

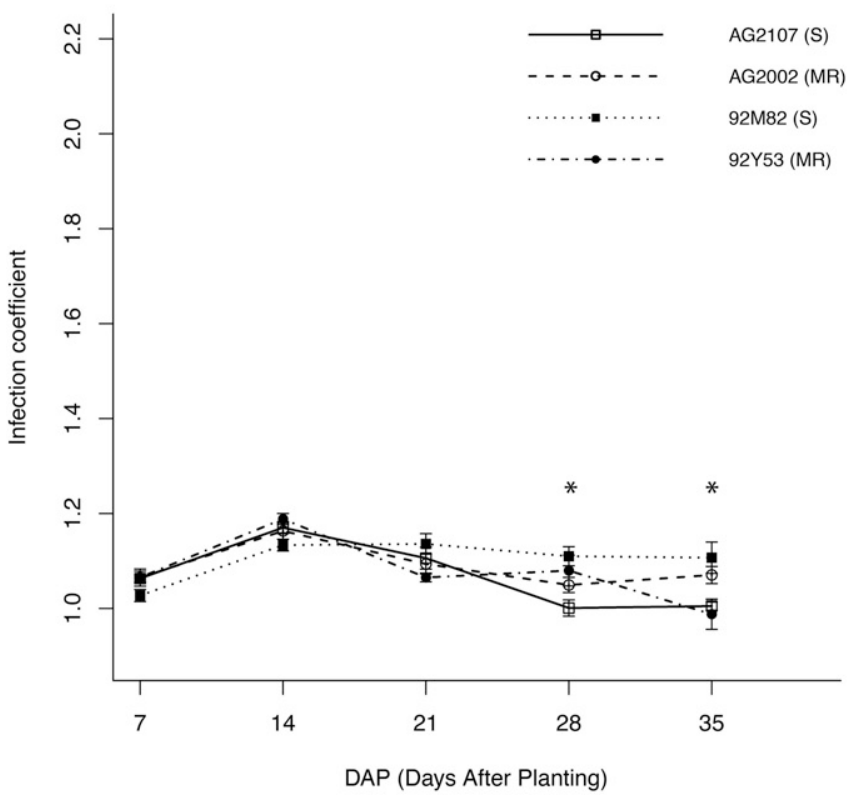

B

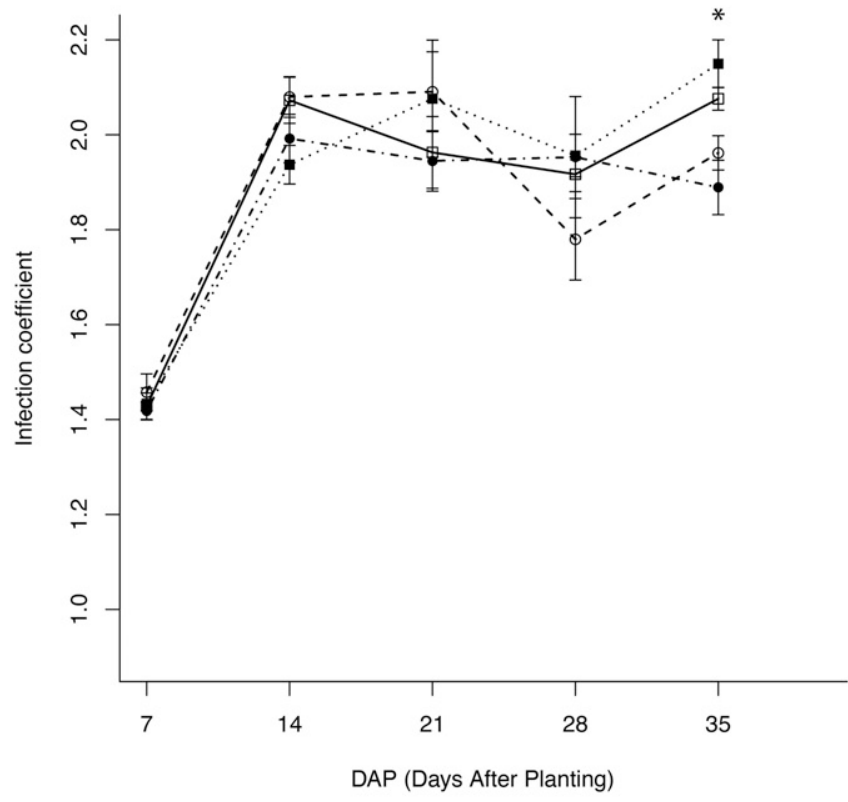

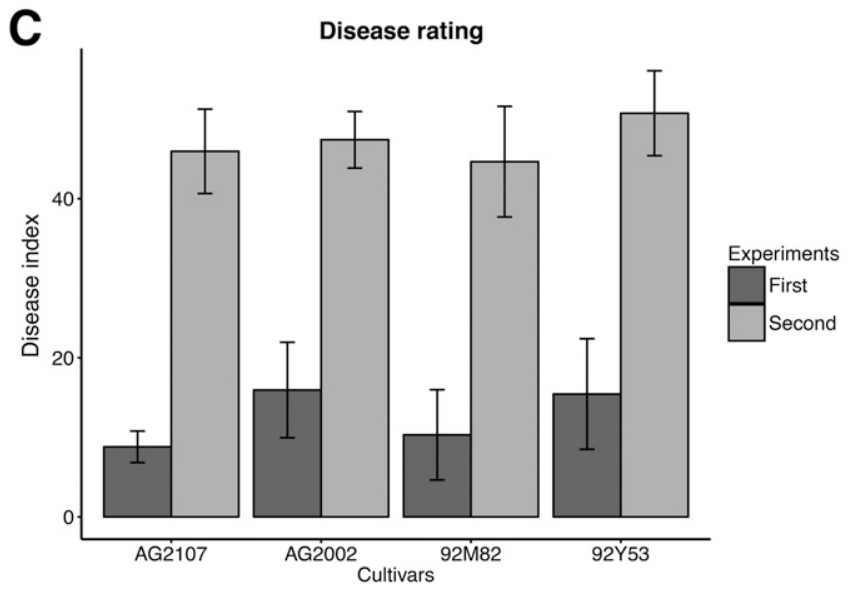

Fig. 1. Temporal dynamics of Fusarium virguliforme infection coefficient over five time points in the first greenhouse experiment (A) and the second greenhouse experiment (B). S indicates susceptible and MR indicates moderate resistance. Disease rating at the fifth sampling time point (35 DAP) for both greenhouse experiments (C). (DX indicates disease index on a scale $0-100$, where 0 indicates healthy plants and 100 indicates most severe sudden death syndrome [SDS] foliar symptoms). Asterisks in A and B indicate significantly different among cultivars $(P<0.05)$. 
detected in root tissue with qPCR as early as the $\mathrm{V} 3$ growth stage for all soybean cultivars, the average $F$. virguliforme IC ranged between 0.91 and 1.03 . The $F$. virguliforme ICs were significantly higher at the East Lansing site than the Decatur site $(P<0.01)$ for the first three sampling time points (growth stages V3, V5, R1). From V5 to R5 growth stage, the $F$. virguliforme IC increased over time at both locations, but the Decatur site had a higher root colonization rate (0.007 IC/day) than the East Lansing site (0.003 IC/day). At R6 growth stage, the $F$. virguliforme IC decreased by 0.060 and 0.079 at the East Lansing site and Decatur site, respectively. After the R6 stage, $F$. virguliforme IC started to increase again and reached maximum colonization level at both field locations postharvest.

At the Decatur site, the first appearance of SDS foliar symptoms was recorded at $40 \mathrm{DAP}$, and gradually developed into most severe foliar symptoms at 99 DAP. The average SDS foliar disease indices were 3.33, 0.56, 63.9, and 0 for soybean cultivars AG2107, AG2002, 92M82, and 92Y53, respectively (Fig. 2C). Despite the significant differences in SDS foliar symptoms among cultivars, $F$. virguliforme IC in roots were not significantly different among cultivars over time. No SDS foliar symptoms were observed at the East Lansing site.
The AUICCs were calculated to evaluate the season-long trends of $F$. virguliforme root colonization. In 2012, AUICCs were not significantly different among the four cultivars at both Decatur $(P=$ 0.08 ) and the East Lansing sites $(P=0.70)$ (Fig. 2A and B). The AUICCs at the Decatur site were significantly higher than at the East Lansing site $(P=0.02$, Supplementary Fig. S3), and SDS foliar symptoms were only observed at the Decatur site (Fig. 2C). The most severe SDS foliar symptoms $(\mathrm{DX}=66.7)$ were present on cultivar 92M82 at the Decatur site. Interestingly, root colonization levels (AUICCs) for 92M82 were lowest among four cultivars (LSD test $P=0.046$, Fig. 2B). The opposite trend was also seen, where cultivars AG2002 and AG2107 showed less severe SDS foliar symptoms (i.e., DX: 0.56 and 3.33) but higher $F$. virguliforme AUICC over the season.

In the 2014 field experiment conducted at the Decatur site, F. virguliforme was detected in all five soybean cultivars, with an average IC ranging between 0.92 and 1.00 as early as 48 DAP. The $F$. virguliforme IC in roots slightly increased at $62 \mathrm{DAP}$, and remained stable until 93 DAP with the average IC ranging between 0.92 and 1.09 (Fig. 3A). F. virguliforme root colonization reached a peak at 107 DAP for all five cultivars. Three out of five cultivars (P92Y11,
A
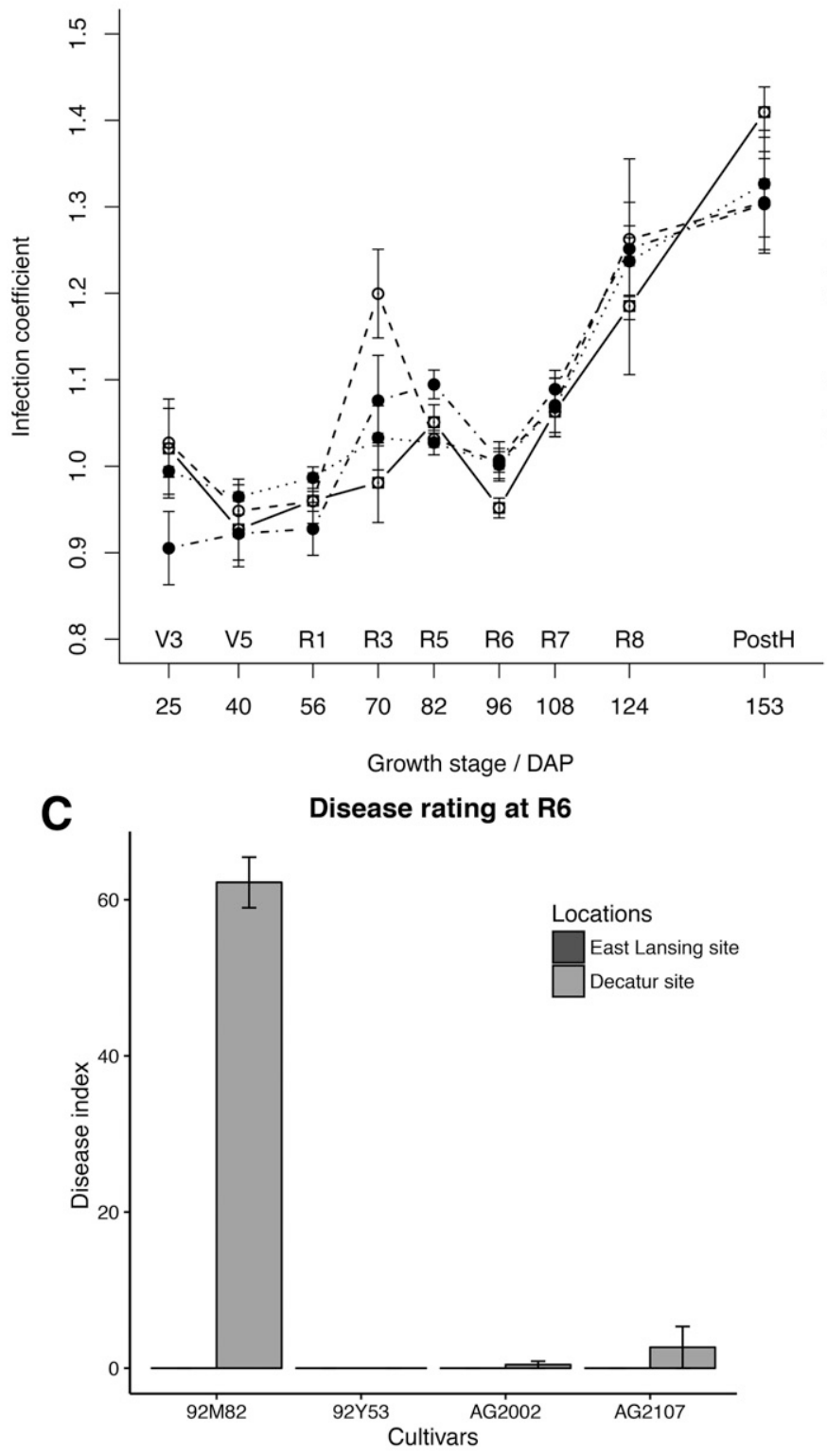

B

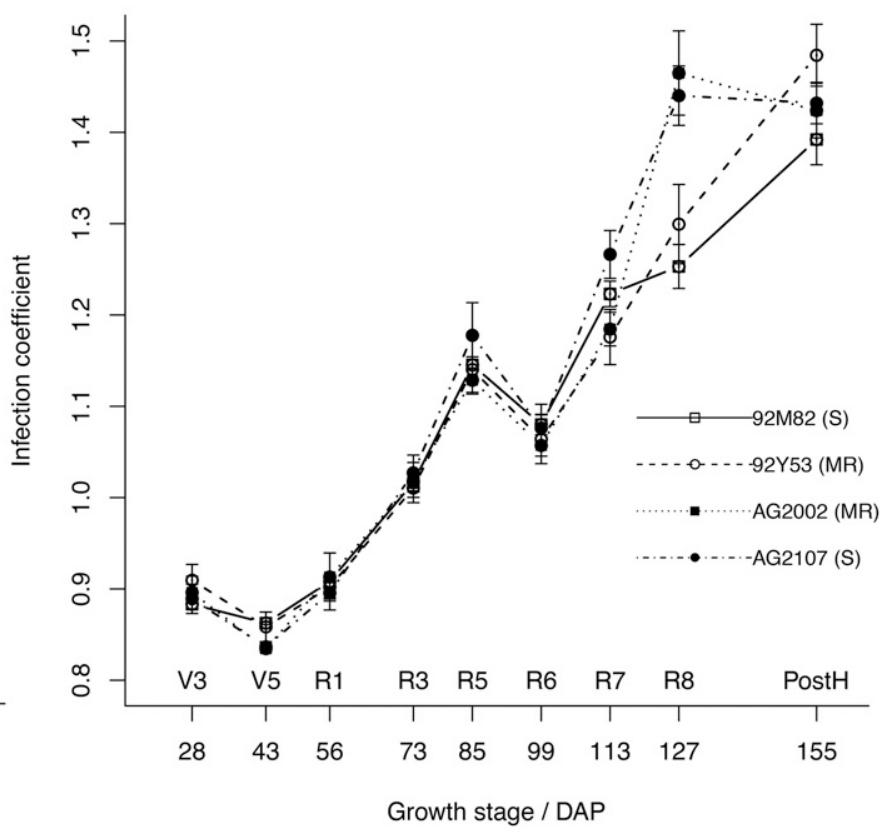

Fig. 2. Temporal dynamics of Fusarium virguliforme infection coefficient over nine sampling points in 2012 at the (A) East Lansing field site and the (B) Decatur field site. (C) Disease ratings at the $\mathrm{R} 6$ stage on all cultivars at both locations. 
P92Y51, and P92Y60) developed SDS foliar symptoms at 93 DAP, while two (P92Y53 and P93M11) did not develop SDS foliar symptoms (Fig. 3B). Although foliar SDS disease indices were significantly different among cultivars $(P=0.0062$, Fig. 3B $)$, the $F$. virguliforme $\mathrm{IC}$ in soybean roots did not significantly correlate with foliar SDS symptoms (Spearman correlation test, $P=0.143$ ).

In 2014, individual plant SDS root severity indices demonstrated a positive, but nonsignificant correlation with the $F$. virguliforme relative quantities in roots $\left(r^{2}=0.02\right.$, Spearman correlation $P=0.21$, Fig. 4A). There was no correlation between individual plant SDS foliar disease indices with $F$. virguliforme ICs in roots $\left(r^{2}<0.001\right.$, Spearman correlation $P=0.80$, Fig. 4B).

\section{Discussion}

The temporal dynamics of $F$. virguliforme in greenhouse and field experiments demonstrated that soybean roots were colonized shortly after planting (germination), with $F$. virguliforme DNA being detected at the earliest sampling points of 7 and 25 DAP for greenhouse and field experiments, respectively. The infection coefficient of $F$. virguliforme increased over time during the trials, and peaked in roots in the field at R7 or R8 sampling points in field experiments. Interestingly, the quantity of $F$. virguliforme DNA did not correlate with soybean susceptibility based on foliar disease ratings. These findings give us an improved understanding of the epidemiology of $F$. virguliforme with disease management implications. A moderately resistant soybean cultivar planted in an infested field may not develop foliar symptoms, but does not guarantee a reduction in $F$. virguliforme inoculum in the soil, and may actually build inoculum pressure. Root rot and root infection may also contribute to hidden yield loss in the absence of obvious foliar SDS symptoms.

$F$. virguliforme root infection and SDS foliar symptoms in the greenhouse. SDS foliar symptom development initializes from root infection, but $F$. virguliforme root infection does not always lead to development of SDS foliar symptoms. SDS foliar symptoms are
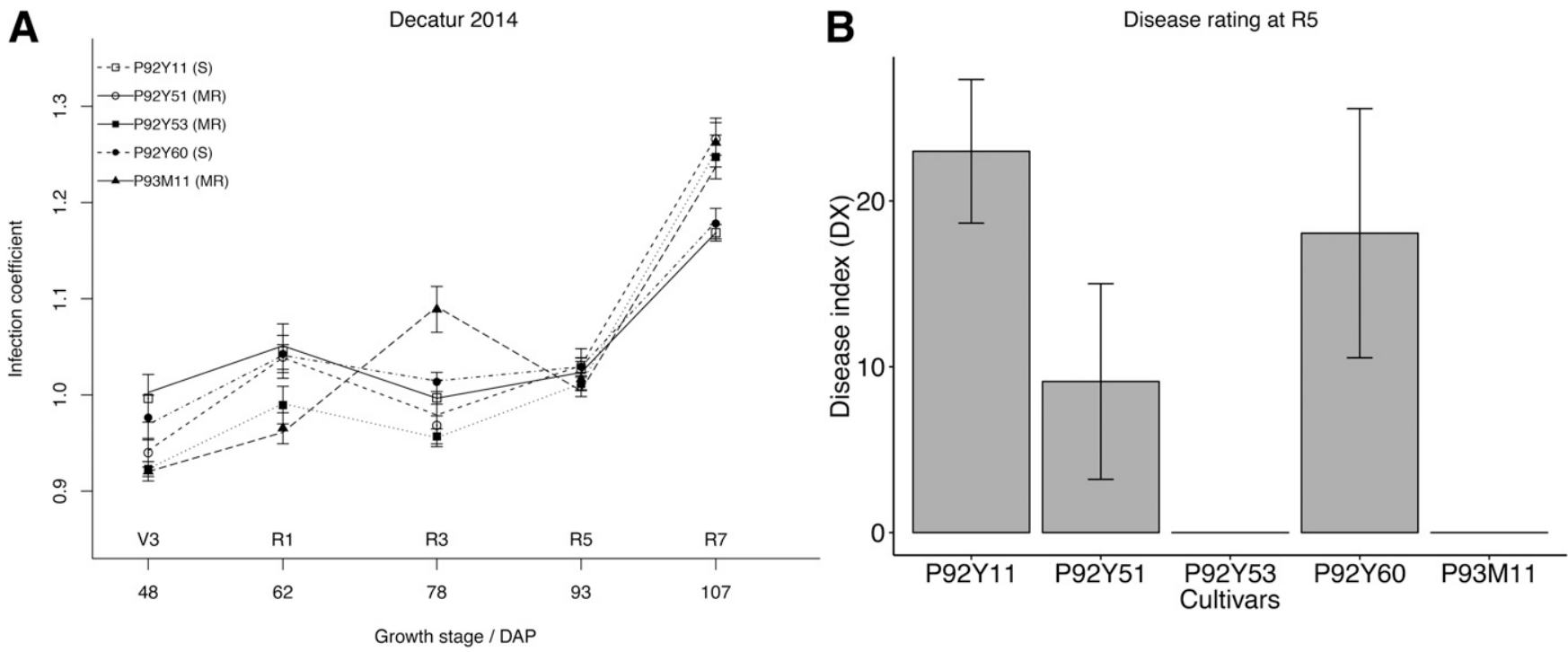

Fig. 3. (A) Temporal dynamics of Fusarium virguliforme in soybean roots over five sampling points at Decatur site in 2014. (B) Sudden death syndrome (SDS) disease rating at R5 stage, disease index was significantly different among cultivars (ANOVA, $P=0.02$ ).
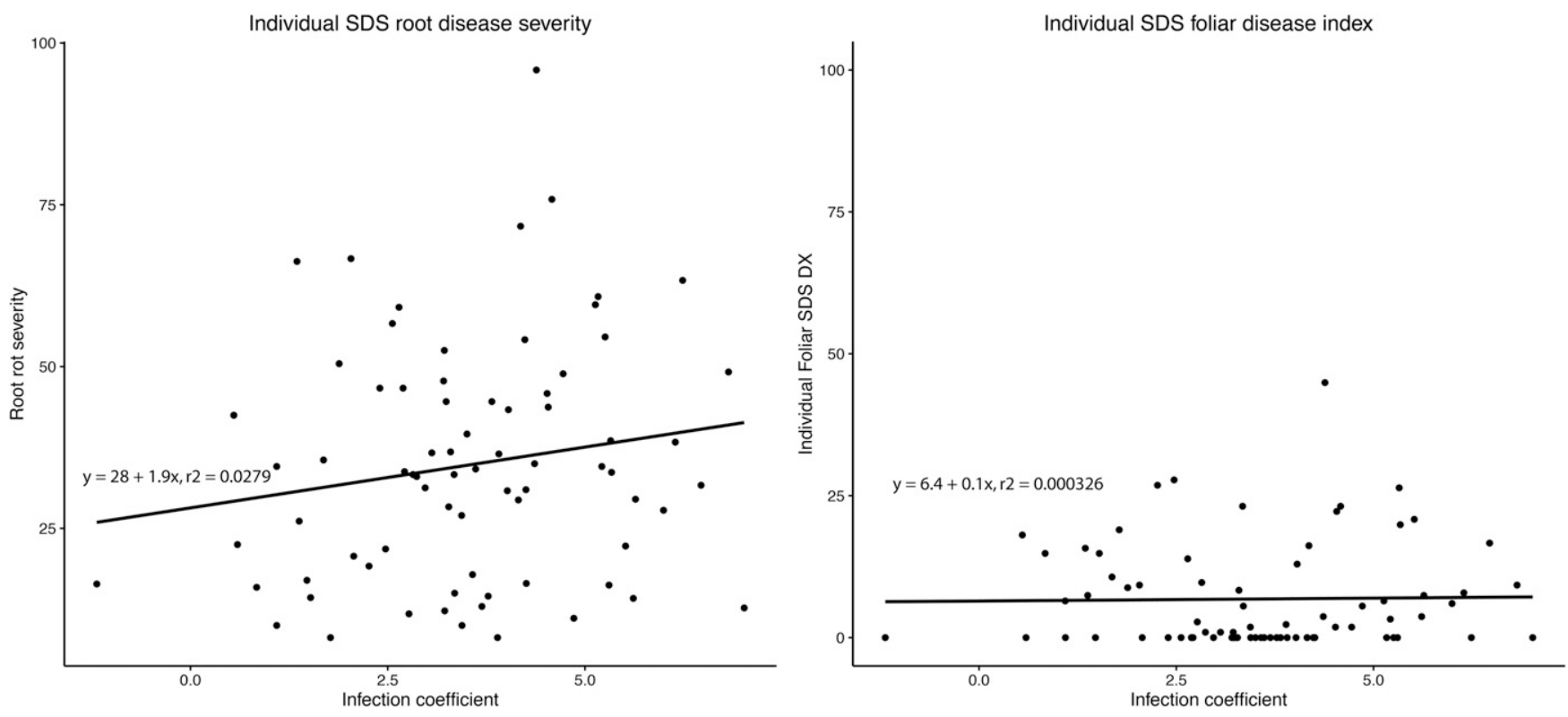

Fig. 4. Individual soybean root samples collected at Decatur site in 2014 at first four time points (i.e., V3, R1, R3, and R5). Correlation between individually rated soybean plants for sudden death syndrome (SDS) symptoms and Fusarium virguliforme infection coefficient in soybean roots. Left: Relation between infection coefficient of $F$. virguliforme and root rot severity percentage rating. Right: Relation between infection coefficient and foliar SDS disease rating index. 
the direct outcome of the soybean leaf programmed cell death that is induced by the toxins produced by $F$. virguliforme in the roots (Chang et al. 2016; Ji et al. 2006). In the greenhouse experiments, SDS symptoms developed on all soybean cultivars with no differences between cultivars, but there were differences in $F$. virguliforme quantity in the roots and foliar disease between the greenhouse experiments.

Both the infection coefficient and the foliar SDS symptoms in the first greenhouse experiment were significantly lower than the second greenhouse experiment (Fig. 1). In addition, the root dry weights were lower in the second greenhouse experiment with higher IC levels and more severe SDS foliar symptoms. The colonization and foliar SDS disease differences in the amount of $F$. virguliforme colonized in the roots between the two greenhouse experiments could be the result of numerous environmental factors, such as soil moisture, temperature, light levels, and different batches of steampasteurized soil. The lack of differences between cultivars within the greenhouse experiments could be due to the high inoculum rate used in the greenhouse experiments compared with in the field. The high inoculum pressure in the greenhouse experiments may have saturated the tolerance for both susceptible and moderately resistant cultivars.

$F$. virguliforme root infection and SDS foliar symptoms in the field. In the 2012 field experiments, we observed contrasting foliar symptoms at each field experimental site. At the East Lansing site, all soybean cultivars were infected with $F$. virguliforme, but no SDS foliar symptoms developed on the four soybean cultivars. Since $F$. virguliforme was detected in the roots, the absence of SDS foliar symptom development may be due to the lack of production or translocation of toxins formed by F. virguliforme. At the Decatur site, with the exact same soybean cultivars planted in 2012, three out of four cultivars exhibited typical SDS foliar symptoms, and again at the Decatur site in 2014 three out of six soybean cultivars developed foliar symptoms. There were four major differences between these two sites that could contribute to the contrasting SDS foliar symptoms: 1) different inoculum type, concentration, and spatial distribution, 2) climatic conditions and irrigation, 3) soil type, and 4) soybean cyst nematode ( $\mathrm{SCN}$ ) pressure.

The Decatur site was a sandy soil naturally infested with $F$. virguliforme, whereas the East Lansing site had a greater clay content and was artificially inoculated. Variation in the ability $F$. virguliforme isolates for root infection and toxin production has been documented (Li et al. 2009). In addition, intraspecific genetic diversity was identified among $F$. virguliforme isolates collected in Michigan, including the naturally infested Decatur location (Wang and Chilvers 2016). The overhead irrigation system at the Decatur location also delivered a much greater volume of water than the drip irrigation system used at the East Lansing site. Combined with the low amount of natural precipitation in 2012 in Michigan, moisture could have been a limiting factor in the lack of SDS foliar symptom development at the East Lansing site (Rupe et al. 1993). In more typical years the use of drip irrigation and artificial inoculation has resulted in high levels of foliar SDS disease levels at the East Lansing location. In addition, the Decatur site has a very high level of SCN pressure, whereas SCN was barely detectable at the East Lansing site. SCN has long been hypothesized to interact with $F$. virguliforme root infection and influence SDS foliar symptom development (Gao et al. 2006; Kandel et al. 2017; Westphal et al. 2014).

Utility of qPCR as a $F$. virguliforme quantification tool. Obtaining accurate and reproducible quantification of $F$. virguliforme from young plants can be difficult using culture based approaches as reported in many previous studies (Luo et al. 1999; Njiti et al. 1997). In this study, $F$. virguliforme was consistently detected using qPCR in all soybean cultivars, even at early sampling time points (V3 growth stage in the field and 7 DAP in the greenhouse). Though no significant differences were detected in $F$. virguliforme quantity among soybean cultivars, the qPCR method provided the necessary detection sensitivity to compare $F$. virguliforme infection at early growth stages. Using culture based approaches, Luo et al. (1999) were able to distinguish resistant and susceptible cultivars in their field study using area under population curve (AUPC). The AUPC method was also adapted in our QPCR data to obtain an overall estimation of $F$. virguliforme root colonization of cultivars over time with the AUICC metric. In our study, AUICCs were not significantly different among soybean cultivars in an ANOVA $(P>0.05$, Supplementary Fig. S4), though SDS foliar symptoms varied among cultivars. For example, cultivar 92M82 had the most severe SDS foliar symptoms in the naturally infested field, but it had the lowest AUICC of the soybean cultivars evaluated (Fig. 2). In contrast, cultivars AG2002 and AG2107 showed slight foliar SDS symptoms, while their roots were highly colonized by $F$. virguliforme as shown in their AUICCs (Fig. 2).

At soybean plant maturity, roots start to degrade due to natural senescence. This senescence was captured in the total root dry weight measurements, and soybean genomic DNA, which both started to decline from the R7 growth stage (Supplementary Fig. S5), with a corresponding increase in the quantity of $F$. virguliforme DNA quantity. The life style of $F$. virguliforme may have switched from a necrotrophic stage into a saprophytic stage for fast colonization, as has been observed in the interaction of Colletotrichum with Arabidopsis thaliana (O'Connell et al. 2012). In addition, maturation group of different soybean cultivars may impact the level of colonization of $F$. virguliforme in roots late in the growing season (Fig. 2). Therefore, root sampling between stages R5 and R7 is recommended to quantify $F$. virguliforme to evaluate for soybean resistance.

Infection coefficient versus absolute quantification. Presentation of qPCR quantification data differs among studies; however, absolute and relative quantification are the two most used measurements. However, methods that determine either absolute or relative quantification can be misleading if the underlying assumptions are not met, resulting in misinterpretation of the data (Bustin et al. 2009). Absolute quantification is widely applied in many qPCR assays for plant-pathogen quantification (Bilodeau et al. 2012; Chilvers et al. 2007; Malvick and Impullitti 2007). The advantage of using absolute quantification is that the results can be directly interpreted as the quantity of the target pathogen. The assumed conditions of the absolute quantification method are that PCR efficiencies between the quantification standard and the unknown samples are within the same range, and that DNA concentration measurement for the quantification standards are accurate. The amplification efficiency of the serially diluted genomic DNA standards is not always consistent with the amplification efficiency of environmental or plant DNA samples, due to the presence of PCR inhibitors in environmental or plant DNA samples. Thus, if PCR efficiency variances are present, the absolute quantification values will not be accurate. Relative quantification of qPCR data for pathogen quantification can help address the presence of PCR inhibitors. The relative quantity of a target pathogen can be presented as $\Delta \mathrm{Ct}$ (Gao et al. 2004; Li et al. 2008) or infection coefficient (Atallah et al. 2007; Valsesia et al. 2005). The advantage of the relative quantification method is that it includes both host (endogenous control) and pathogen (target) components, which provide pathogen quantification in the context of pathogen colonization within the plant host. The assumptions for valid relative quantification are to have equal PCR efficiencies for both plant host and pathogen qPCR assays (Livak and Schmittgen 2001). There are advantages and disadvantages of using either of these two quantifications methods. In this study, relative quantification, calculated by determining both plant and pathogen $\mathrm{Ct}$ values, was used to evaluate the $F$. virguliforme quantity in soybean roots. The PCR efficiencies between the $F$. virguliforme and soybean $\mathrm{qPCR}$ assays were within the range of 90 to $95 \%$. One possible limitation of the qPCR-based quantification method is that dead and live pathogen cells present in roots can both be quantified in the qPCR, possibly resulting in overestimation of actively pathogenic $F$. virguliforme in soybean roots. For large-scale screening of $F$. virguliforme root colonization in a breeding project, the absolute quantification can be used to increase the processing throughput and reduce reagent costs.

Conclusions. In conclusion, both greenhouse and field experiments demonstrated that the appearance and severity of SDS foliar symptoms are not always dependent on the relative quantity of 
F. virguliforme inside colonized soybean plants. Interestingly, we were not able to identify a specific IC related to development of foliar disease symptoms in the greenhouse or field experiments, although there were correlations between the level of IC and severity of symptoms in greenhouse and field experiments. This highlights the difficulty in screening germplasm for SDS resistance. Additional experiments may need to consider the location (tap versus lateral roots) and type (cortical versus vascular) of root infection. Since SDS starts with root infection, a wider range of soybean germplasm should be screened for root infection resistance. Due to the focus of this study, four soybean cultivars were selected to test the temporal dynamics of $F$. virguliforme in soybean roots. Though no significant differences in ICs were found among cultivars, it does not necessarily translate to no significant difference among cultivars if more soybean germplasm is included. We demonstrated an efficient and accurate method for quantification of $F$. virguliforme in soybean roots, which can potentially be used in a breeding program to phenotype soybean germplasm for resistance to $F$. virguliforme root infection at both early and late soybean growth stages.

\section{Acknowledgments}

We thank Adam Byrne and John Boyse for their assistance with fieldwork, Dr. Carl and Amy Druskovich for land use, and Dr. Jan Byrne for her excellent editorial suggestions.

\section{Literature Cited}

Abeysekara, N. S., and Bhattacharyya, M. K. 2014. Analyses of the xylem sap proteomes identified candidate Fusarium virguliforme proteinacious toxins. PLoS One 9:e93667.

Aoki, T., O'Donnell, K., Homma, Y., and Lattanzi, A. R. 2003. Sudden-death syndrome of soybean is caused by two morphologically and phylogenetically distinct species within the Fusarium solani species complex-F. virguliforme in North America and F. tucumaniae in South America. Mycologia 95:660-684.

Arias, M. M. D., Leandro, L. F., and Munkvold, G. P. 2013. Aggressiveness of Fusarium species and impact of root infection on growth and yield of soybeans. Phytopathology 103:822-832.

Atallah, Z. K., Bae, J., Jansky, S. H., Rouse, D. I., and Stevenson, W. R. 2007. Multiplex real-time quantitative PCR to detect and quantify Verticillium dahliae colonization in potato lines that differ in response to Verticillium wilt. Phytopathology 97:865-872

Bilodeau, G. J., Koike, S. T., Uribe, P., and Martin, F. N. 2012. Development of an assay for rapid detection and quantification of Verticillium dahliae in soil. Phytopathology 102:331-343.

Bustin, S. A., Benes, V., Garson, J. A., Hellemans, J., Huggett, J., Kubista, M., Mueller, R., Nolan, T., Pfaffl, M. W., Shipley, G. L., Vandesompele, J., and Wittwer, C. T. 2009. The MIQE guidelines: Minimum information for publication of quantitative real-time PCR experiments. Clin. Chem. 55:611-622.

Chang, H. X., Domier, L. L., Radwan, O., Yendrek, C. R., Hudson, M. E., and Hartman, G. L. 2016. Identification of multiple phytotoxins produced by Fusarium virguliforme including a phytotoxic effector (FvNIS1) associated with sudden death syndrome foliar symptoms. Mol. Plant-Microbe Interact. 29:96-108.

Chilvers, M. I., du Toit, L. J., Akamatsu, H., and Peever, T. L. 2007. A real-time, quantitative PCR seed assay for Botrytis spp. that cause neck rot of onion. Plant Dis. 91:599-608.

Cho, J. H., Rupe, J. C., Cummings, M. S., and Gbur, E. E. 2001. Isolation and identification of Fusarium solani $\mathrm{f}$. sp. glycines from soil on modified Nash and Snyder's medium. Plant Dis. 85:256-260.

Clark, W. D., Reyes-Valdes, M. H., Bond, J., and Kantartzi, S. K. 2013. Performance of LS97-1610x'Spencer' soybean recombinant inbred line population segregating for resistance to Fusarium virguliforme. Can. J. Plant Sci. 93:1179-1185

De Mendiburu, F. 2014. Agricolae: Statistical procedures for agricultural research. $\mathrm{R}$ package version 1:1-6.

Dorrance, A. E., Kleinhenz, M. D., McClure, S. A., and Tuttle, N. T. 2003. Temperature, moisture, and seed treatment effects on Rhizoctonia solani root rot of soybean. Plant Dis. 87:533-538.

Fehr, W., Caviness, C., Burmood, D., and Pennington, J. 1971. Stage of development descriptions for soybeans, Glycine max (L.) Merrill. Crop Sci. 11:929-931.

Gao, X., Hartman, G., and Niblack, T. 2006. Early infection of soybean roots by Fusarium solani f. sp. glycines. Phytopathology 96:S38

Gao, X., Jackson, T. A., Lambert, K. N., Li, S., Hartman, G. L., and Niblack, T. L. 2004. Detection and quantification of Fusarium solani f. sp. glycines in soybean roots with real-time quantitative polymerase chain reaction. Plant Dis. 88:1372-1380.

Gibson, P., Shenaut, M., Njiti, V., Suttner, R., and Myers, O., Jr. 1994. Soybean varietal response to sudden death syndrome. Pages 6-7 in: Proc. 24th Soybean Seed Res Conf., Chicago, Illinois.
Gongora-Canul, C., Nutter, F. W., Jr., and Leandro, L. F. S. 2012. Temporal dynamics of root and foliar severity of soybean sudden death syndrome at different inoculum densities. Eur. J. Plant Pathol. 132:71-79.

Gongora-Canul, C. C., and Leandro, L. F. S. 2011a. Plant age affects root infection and development of foliar symptoms of soybean sudden death syndrome. Plant Dis. 95:242-247.

Gongora-Canul, C. C., and Leandro, L. F. S. 2011b. Effect of soil temperature and plant age at time of inoculation on progress of root rot and foliar symptoms of soybean sudden death syndrome. Plant Dis. 95:436-440.

Haudenshield, J. S., and Hartman, G. L. 2011. Exogenous controls increase negative call veracity in multiplexed, quantitative PCR assays for Phakopsora pachyrhizi. Plant Dis. 95:343-352.

Hershman, D. E., Hendrix, J. W., Stuckey, R. E., Bachi, P. R., and Henson, G 1990. Influence of planting date and cultivar on soybean sudden death syndrome in Kentucky. Plant Dis. 74:761-766.

Huang, Y. H., and Hartman, G. L. 1998. Reaction of selected soybean genotypes to isolates of Fusarium solani f. sp. glycines and their culture filtrates. Plant Dis. 82:999-1002.

Hughes, T. J., Atallah, Z. K., and Grau, C. R. 2009. Real-time PCR assays for the quantification of Phialophora gregata $\mathrm{f}$. sp. sojae IGS genotypes A and B. Phytopathology 99:1008-1014.

Ji, J., Scott, M. P., and Bhattacharyya, M. K. 2006. Light is essential for degradation of ribulose-1.5-bisphosphate carboxylase-oxygenase large subunit during sudden death syndrome development in soybean. Plant Biol. 8:597-605.

Kandel, Y. R., Haudenshield, J., Srour, A. Y., Islam, K. T., Fakhoury, A. M., Santos, P., Wang, J., Chilvers, M. I., Hartman, G., Malvick, D., Floyd, C. M., Mueller, D., and Leandro, L. 2015. Multi-laboratory comparison of quantitative PCR assays for detection and quantification of Fusarium virguliforme from soybean roots and soil. Phytopathology 105:1601-1611.

Kandel, Y. R., Wise, K. A., Bradley, C. A., Chilvers, M. I., Byrne, A. M., Tenuta, A. U., Faghihi, J., Wiggs, S. N., Tylka, G. L., and Mueller, D. S. 2017. Effect of SCN resistance source and seed treatment on population densities of Heterodera glycines, sudden death syndrome, and yield of soybean. Plant Dis. 101:2137-2143.

Kandel, Y. R., Wise, K. A., Bradley, C. A., Tenuta, A. U., and Mueller, D. S. 2016 Effect of planting date, seed treatment, and cultivar on plant population, sudden death syndrome, and yield of soybean. Plant Dis. 100:1735-1743.

Kazi, S., Shultz, J., Afzal, J., Johnson, J., Njiti, V. N., and Lightfoot, D. A. 2008 Separate loci underlie resistance to root infection and leaf scorch during soybean sudden death syndrome. Theor. Appl. Genet. 116:967-977.

Leslie, J. F., and Summerell, B. A. 2008. The Fusarium Laboratory Manual. Wiley, Hoboken, NJ.

Li, S., Hartman, G. L., and Chen, Y. 2009. Evaluation of aggressiveness of Fusarium virguliforme isolates that cause soybean sudden death. J. Plant Pathol. 91:77-86.

Li, S., Hartman, G. L., Domier, L. L., and Boykin, D. 2008. Quantification of Fusarium solani f. sp. glycines isolates in soybean roots by colony-forming unit assays and real-time quantitative PCR. Theor. Appl. Genet. 117:343-352.

Livak, K. J., and Schmittgen, T. D. 2001. Analysis of relative gene expression data using real-time quantitative PCR and the 2(T)(-Delta Delta CT) method. Methods 25:402-408.

Luo, Y., Myers, O., Lightfoot, D., and Schmidt, M. 1999. Root colonization of soybean cultivars in the field by Fusarium solani f. sp. glycines. Plant Dis. 83:1155-1159.

Malvick, D., and Impullitti, A. 2007. Detection and quantification of Phialophora gregata in soybean and soil samples with a quantitative, real-time PCR assay. Plant Dis. 91:736-742.

Navi, S. S., and Yang, X. 2008. Foliar symptom expression in association with early infection and xylem colonization by Fusarium virguliforme (formerly F. solani f. sp. glycines), the causal agent of soybean sudden death syndrome. Plant Health Prog. doi:10.1094/PHP-2008-0222-01-RS.

Njiti, V. N., Shenaut, M. A., Suttner, R. J., Schmidt, M. E., and Gibson, P. T. 1996 Soybean response to sudden death syndrome: Inheritance influenced by cyst nematode resistance in Pyramid x Douglas progenies. Crop Sci. 36:1165-1170.

Njiti, V. N., Suttner, R. J., Gray, L. E., Gibson, P. T., and Lightfoot, D. A. 1997. Rate-reducing resistance to Fusarium solani f. sp. phaseoli underlies field resistance to soybean sudden death syndrome. Crop Sci. 37:132-138

O'Connell, R. J., Thon, M. R., Hacquard, S., Amyotte, S. G., Kleemann, J., Torres, M. F., Damm, U., Buiate, E. A., Epstein, L., Alkan, N., Altmuller, J., AlvaradoBalderrama, L., Bauser, C. A., Becker, C., Birren, B. W., Chen, Z. H., Choi, J., Crouch, J. A., Duvick, J. P., Farman, M. A., Gan, P., Heiman, D., Henrissat, B., Howard, R. J., Kabbage, M., Koch, C., Kracher, B., Kubo, Y., Law, A. D., Lebrun, M. H., Lee, Y. H., Miyara, I., Moore, N., Neumann, U., Nordstrom, K., Panaccione, D. G., Panstruga, R., Place, M., Proctor, R. H., Prusky, D., Rech, G., Reinhardt, R., Rollins, J. A., Rounsley, S., Schardl, C. L., Schwartz, D. C., Shenoy, N., Shirasu, K., Sikhakolli, U. R., Stuber, K. Sukno, S. A., Sweigard, J. A., Takano, Y., Takahara, H., Trail, F., van der Does, H. C., Voll, L. M., Will, I., Young, S., Zeng, Q. D., Zhang, J. Z., Zhou, S. G., Dickman, M. B., Schulze-Lefert, P., van Themaat, E. V. L., Ma, L. J., and Vaillancourt, L. J. 2012. Lifestyle transitions in plant pathogenic Colletotrichum fungi deciphered by genome and transcriptome analyses. Nat. Genet. 44:1060-1065. 
Ortiz-Ribbing, L. M., and Eastburn, D. M. 2004. Soybean root systems and sudden death syndrome severity: Taproot and lateral root infection. Plant Dis. 88: 1011-1016.

R Core Team. 2015. R: A Language and Environment for Statistical Computing. R Foundation for Statistical Computing, Vienna, Austria.

Roy, K. W., Hershman, D. E., Rupe, J. C., and Abney, T. S. 1997. Sudden death syndrome of soybean. Plant Dis. 81:1100-1111.

Rupe, J. C. 1989. Frequency and pathogenicity of Fusarium solani recovered from soybeans with sudden death syndrome. Plant Dis. 73:581-584.

Rupe, J. C., Sabbe, W. E., Robbins, R. T., and Gbur, E. E. 1993. Soil and plant factors associated with sudden-death syndrome of soybean. J. Prod. Agric. 6:218-221.

Scherm, H., and Yang, X. B. 1996. Development of sudden death syndrome of soybean in relation to soil temperature and soil water matric potential. Phytopathology 86:642-649.

Srour, A., Afzal, A. J., Blahut-Beatty, L., Hemmati, N., Simmonds, D. H., Li, W., Liu, M., Town, C. D., Sharma, H., Arelli, P., and Lightfoot, D. A. 2012. The receptor like kinase at Rhg1-a/Rfs2 caused pleiotropic resistance to sudden death syndrome and soybean cyst nematode as a transgene by altering signaling responses. BMC Genomics 13:368.
Valsesia, G., Gobbin, D., Patocchi, A., Vecchione, A., Pertot, I., and Gessler, C. 2005. Development of a high-throughput method for quantification of Plasmopara viticola DNA in grapevine leaves by means of quantitative realtime polymerase chain reaction. Phytopathology 95:672-678.

Wang, J., and Chilvers, M. I. 2016. Development and characterization of microsatellite markers for Fusarium virguliforme and their transferability to clade 2 of the Fusarium solani species complex. Fungal Ecol. 20:7-14.

Wang, J., Jacobs, J. L., Byrne, J. M., and Chilvers, M. I. 2015. Improved diagnoses and quantification of Fusarium virguliforme, causal agent of soybean sudden death syndrome. Phytopathology 105:378-387.

Wen, Z., Tan, R., Yuan, J., Bales, C., Du, W., Zhang, S., Chilvers, M. I., Schmidt C., Song, Q., Cregan, P. B., and Wang, D. 2014. Genome-wide association mapping of quantitative resistance to sudden death syndrome in soybean. BMC Genomics 15:809.

Westphal, A., Li, C., Xing, L., McKay, A., and Malvick, D. 2014. Contributions of Fusarium virguliforme and Heterodera glycines to the disease complex of sudden death syndrome of soybean. PLoS One 9:e99529.

Wickham, H. 2009. GGplot2: Elegant Graphics for Data Analysis. Springer, New York. 